effect of ethanol on the development of systemic lupus erythematosus (SLE) remains controversial. This study was performed to determine the potential role of moderate ethanol consumption in SLE pathological progression and clarify its functional mechanism.

Methods We used MRL/lpr mice to assess whether ethanol drinking has any impact on the development of SLE and investigated whether ethanol regulates pathologic progression of SLE through inhibiting lipid rafts.

Results We found that $10 \%$ ethanol in vivo delayed disease progression and organ damage and prolonged survival. In vitro ethanol treatment not only inhibited the aggregation, proliferation, adhesion molecule expression and IFN- $\gamma$ secretion of $\mathrm{T}$ cells, but also decreased lipid raft clustering on $\mathrm{T}$ cells. In addition, ethanol inhibited SLE serum-induced skin inflammation and monocyte differentiation into dendritic cells (DCs). Furthermore, ethanol treatment of monocytes that were in the process of differentiating into DCs decreased lipid raft clustering.

Conclusions These data strongly support the viewpoint that ethanol delays the disease progression of SLE by inhibiting lipid raft clustering and suggest that moderate drinking of ethanol may have a protective value for patients with SLE.

\section{BIIB059, A MONOCLONAL ANTIBODY TARGETING BDCA2, DEMONSTRATES EVIDENCE OF PROOF OF BIOLOGICAL ACTIVITY IN SUBJECTS WITH CUTANEOUS LUPUS}

${ }^{1} \mathrm{R}$ Furie ${ }^{*},{ }^{2} \mathrm{VP}$ Werth, ${ }^{3} \mathrm{JF}$ Merola, ${ }^{4} \mathrm{~W}$ Wang, ${ }^{5} \mathrm{D}$ Rabah, ${ }^{6} \mathrm{C}$ Barbey, ${ }^{7} \mathrm{~K}$ Smirnakis, ${ }^{8} \mathrm{~B}$ Werneburg, ${ }^{9} \mathrm{~J}$ Bornstein, TL Reynolds ${ }^{10},{ }^{11} \mathrm{~L}$ Stevenson, ${ }^{12} \mathrm{~N}$ Franchimont. ${ }^{1}$ Northwell Health, Division of Rheumatology, Great Neck NewYork, USA; ${ }^{2}$ University of Pennsylvania School of Medicine, Department of Dermatology, Philadelphia, Pennsylvania, USA; ${ }^{3}$ Harvard Medical School, Centre for Skin and Related Musculoskeletal Diseases, Boston, Massachusetts, USA; ${ }^{4}$ Biogen, Biostatistics, Cambridge, Massachusetts, USA; ${ }^{5}$ Biogen, Research/Immuno Discovery Biology, Cambridge, Massachusetts, USA; ${ }^{6}$ Biogen, Biosimilars Medical/Affiliate Medical Affairs/Neurology, Cambridge, Massachusetts, USA; 'Biogen, Drug Safety and Benefit Risk Management, Cambridge, Massachusetts, USA; ${ }^{8}$ Biogen, US Medical/Global Medical Affairs Neurology, Cambridge, Massachusetts, USA; ${ }^{9}$ Biogen, Clinical Development/Immunology Drug Innovation Unit, Cambridge, Massachusetts, USA; ${ }^{10}$ Biogen, Research/In-Vitro Sciences, Cambridge, Massachusetts, USA; ${ }^{11}$ Biogen, Translational Sciences/Biomolecular and Small Molecule Science, Cambridge, Massachusetts, USA; ${ }^{12}$ Biogen, Immunology Drug Innovation Unit, Cambridge, Massachusetts, USA

\subsection{6/lupus-2017-000215.83}

Background and aims Type I interferons (IFN-I) are central to the pathogenesis of systemic lupus erythematosus (SLE). BDCA2 is a plasmacytoid dendritic cell (pDC)-specific receptor that, upon engagement, inhibits the production of IFN-I and other inflammatory mediators. In this first-in-patient phase $1 \mathrm{~b}$ study, biological activity of BIIB059, a humanised anti-BDCA2 monoclonal antibody, was evaluated in SLE subjects with active cutaneous lupus (CLE).

Methods 12 adult SLE subjects with active CLE received a single IV administration of either BIIB059 $20 \mathrm{mg} / \mathrm{kg} \quad(\mathrm{n}=8)$ or placebo $(n=4)$. A panel of IFN-responsive genes (IRG) was assessed from whole blood. Cellular infiltration and expression of MxA and IFITM3 were evaluated in skin biopsies from active lesions at baseline and week 4. CLE disease activity was determined using the Cutaneous Lupus Erythematosus Disease Area and Severity Index (CLASI). Safety data were also collected.

Results BIIB059 decreased the expression of IRG in blood and MxA and IFITM3 proteins in skin. CD45+ cells were reduced in skin biopsies of BIIB059-treated subjects. The reduction in inflammatory cells as well as MxA and IFITM3 expression at week 4 correlated with improvement in CLASI activity score at week 12 . BIIB059 was well tolerated with no withdrawals due to AEs.

Conclusions The study, confirming the major role played by pDCs in the production of IFN-I in the blood and skin in CLE, supports further development of BIIB059.

\section{PROSPECTIVE SINGLE CENTRE STUDY OF EFFECTIVENESS OF UPFRONT RITUXIMAB AND MYCOPHENOLATE WITH MINIMUM STEROID IN SLE}

BG Dharmanand S C*. SAKRA World Hospital, Rheumatology and autoimmune diseases, Bangalore, India

\subsection{6/lupus-2017-000215.84}

Background and aims Treatment options for SLE have significant morbidity and mortality. Side effects from corticosteroid usage limit patient adherence and treatment efficacy. B cell depletion appears to target a critical pathophysiological pathway in SLE. Trails with rituximab has shown mixed results.

We aim to analyse our experience of using rituximab and mycophenolate upfront on presentation with minimum oral steroids.

Methods 12 patients with SLE, seen between Jan 2015 to march 2016, were included in the study. All patients completed 6 months of follow-up. Patients were treated with 2 doses of rituximab (1 g) and methyl prednisolone $(500 \mathrm{mg}$ ) on days 1 and 15 , and maintenance treatment of mycophenolate mofetil $(2000 \mathrm{mg})$ and low dose prednisolone $(<7.5 \mathrm{mg})$ which was tapered off.

Results 10 were females and 2 males. Mean age of the patients is 24.5. 9 had lupus nephritis, 1 mesenteric vasculitis, 1 CNS vasculitis and 1 severe cutaneous vasculitis with pancytopenia. Average SLEDAI improved from 14 to 4 . 6/9 LN attained complete renal remission and 2 partial remission. one patient died due to infection and renal disease 15 days after infusion. 2 vasculitis and one NPSLE patient improved completely. Two patients had infection requiring hospitalisation with in 8 weeks of infusion and one patient had severe bradycardia during the infusion and received only $1000 \mathrm{mg}$ rituximab. Steroid was stopped by 6 months in 6 patients and in the dose was below $5 \mathrm{mg}$ in rest.

Conclusions Early Rituximab and mycophenolate is an effective option for treating severe lupus and has steroid sparing property.

\section{SAFETY OF BELIMUMAB IN PATIENTS WITH SYSTEMIC LUPUS ERYTHEMATOSUS IN CLINICAL PRACTICE SETTING}

${ }^{1} \mathrm{~N}$ Duque, ${ }^{1} \mathrm{M}$ Saldarriaga, ${ }^{1} \mathrm{~L}$ Uribe, ${ }^{2,3} \mathrm{LA}$ González, ${ }^{2} \mathrm{OJ}$ Felipe, ${ }^{2} \mathrm{C}$ Cerón, ${ }^{1} \mathrm{~A}$ Uribe, 1,3JA Gómez-Puerta*. 'Medicarte IPS, Area del conocimiento, Medellin, Colombia; ${ }^{2}$ Medicarte IPS, Reumatología, Medellin, Colombia; ${ }^{3}$ Universidad de Antioquia, Grupo de Reumatología, Medellín, Colombia

\subsection{6/lupus-2017-000215.85}

Background and aims Clinical trials have demonstrated a safety profile of belimumab in SLE patients. Safety of belimumab under daily clinical practice is less well known. Our objective 